\title{
On the Average Spectral Efficiency of Interference-Limited Full-Duplex Networks
}

\author{
(Invited Paper)
}

\author{
Hirley Alves*†, Carlos H. M. de Lima* ${ }^{* \ddagger}$, Pedro H. J. Nardelli*, Richard Demo Souza ${ }^{\dagger}$ and Matti Latva-aho* \\ ${ }^{*}$ Centre for Wireless Communications (CWC), University of Oulu, Finland \\ $\dagger$ Federal University of Technology - Paraná (UTFPR), Curitiba, Brazil \\ ¥ Universidade Estadual Paulista (UNESP), São João da Boa Vista, Brazil \\ \{halves, nardelli,matla\}@ee.oulu.fi, carlos.lima@sjbv.unesp.br, richard@utfpr.edu.br
}

\begin{abstract}
This paper studies how dense deployments of small cells with full duplex technology perform under various network configurations and channel conditions. The resulting interference at the receiver of interest combines both its intrinsic self-interference and components from co-channel base stations and user equipment transmissions. Network deployment is represented by a Poisson field of transmitters, while a composite channel with log-normal shadowing and Nakagami- $m$ fading describes our propagation model. Herein, a stochastic geometry framework is used to first characterize the interference profiles for the full duplex scenarios under consideration, and then derive closed-form expressions for the Average Spectral Efficiency (ASE). Results show that the Self-Interference (SI) dominates the aggregate interference component and Full-Duplex (FD) networks outperform HalfDuplex (HD) networks in terms of ASE for SI cancellation values lower than $-70 \mathrm{~dB}$.
\end{abstract}

\section{INTRODUCTION}

By 2020, a myriad of connected devices will change the way we live, make business, gather into social groups and interact. As a result, the next generation of mobile access networks will experience a thousand-fold increase in traffic volume compared to today's standards, which will require new technologies and practices as well as a rearrangement of the spectrum.

In this context Full-Duplex (FD) communication appears as a promising spectrally efficient technology, since, theoretical FD systems can double the overall spectral efficiency when compared to the traditional Half-Duplex (HD) networks [1]-[3]. In a FD network, devices are able to transmit and receive simultaneously and in the same frequency band, which brings high spectral efficiency for the upcoming wireless communication systems [1]-[6]. However, in practice such gain is not attainable once FD nodes suffer from Self-Interference (SI) and extra CoChannel Interference (CCI) from other concurrent (sametype) transmitters [7], therefore its performance is severely deteriorated. Nevertheless, due to such potential, FD communications have gained considerable attention and several papers appeared recently with promising signal processing techniques for SI interference mitigation [1], [2], [5], [6]. These works show that even though ideal FD operation is not yet attainable, practical FD communications are feasible once SI is considerably attenuated. As pointed out in [1]-[4] the extent of these gains achieved by FD nodes depends on the SI cancellation level. Moreover, SI can be modeled as a fading channel which allows the emulation of various (non) line-of-sight configurations [1]-[3].

So far, most of the publications address the achievable rate from a information theoretic point of view, or solutions to cope with the SI in single cell deployment scenarios [1][6]. However, system-level evaluation of the large scale deployments of FD communicating nodes has received very little attention. Therefore, in this paper we investigate the system-level performance of interference limited FD networks under composite fading channel, which encompass both fading and shadowing. Such composite fading channel allows one to evaluate a large number of channel configurations (e.g. severity of the fading and shadowing, presence or not of line of sight) and to do so we resort to a mathematical framework based on stochastic geometry. Moreover, we derive closed-form expressions for the Signal-to-Interference Ratio (SIR) and the Average Spectral Efficiency (ASE) of FD networks. Our results show that users in full-duplex mode are capable of achieving higher rates when compared to HD networks, even though subject to higher levels of interference since a FD node perceives besides its own SI additional CCI from adjacent cells.

The reminder of this paper is organized as follows: Section II presents the propagation and deployment models and introduces the mathematical framework. Section III introduces the interference models as well as the ASE metric, while Section IV presents some significant numerical results. Finally, Section V concludes the paper.

\section{System Model AND ANALYTIC FrAmEWORK}

Consider an interference-limited network where all nodes operate in FD fashion. Therefore, the devices are 
able to transmit and receive simultaneously at a cost of SI. Consider that Base Stations (BSs) and User Equipments (UEs) have dedicated antennas for transmission and reception. Even though employing advanced interference cancellation and antenna attenuation techniques, there still remains a residual self-interference level [1]-[3], which can be modeled as a fading channel that allows one to emulate various (non) line-of-sight configurations [1], [3]. Bearing this in mind and in order to account for extensive number of fading settings, we assume that all channel coefficients are quasi-static and follow a composite fading channel distribution, which is composed of LogNormal (LN) shadowing and Nakagami- $m$ fading. The Downlink (DL) of a traditional HD network constitutes our benchmark scenario wherein the user of interest is interfered by surrounding small cells. BSs independently schedule a random user in every transmission interval. All communicating nodes are equipped with omni-directional antennas. BSs and UEs are also assumed to have full buffer and symmetric traffic patterns.

\section{A. Propagation and Network Deployment Models}

Radio links are affected by path-loss, large-scale shadowing and multi-path fading which are assumed to be mutually independent and multiplicative phenomena [8]. The received power at the user of interest $u_{0}$ from an arbitrary transmitter $b_{i}$ located $r_{i 0}$ meters away is

$$
Y_{i 0}=p_{i 0} r_{i 0}^{-\alpha} x_{i 0}
$$

where $p_{i 0}$ is the transmit power, $\alpha$ is the path-loss exponent and $x_{i 0}$ represents the squared-envelop of composite fading channel. The composite distribution of the received squared-envelop due to LN shadowing and Nakagami$m$ fading has a Gamma-LN distribution with Probability Density Function (PDF)

$$
\begin{aligned}
f_{X}(x) & =\int_{0}^{\infty}\left(\frac{m}{\omega}\right)^{m} \frac{x^{m-1}}{\Gamma(m)} \exp \left(-\frac{m}{\omega} x\right) \\
& \times \frac{\xi}{\sqrt{2 \pi} \sigma \omega} \exp \left[-\frac{\left(\xi \ln \omega-\mu_{\Omega_{p}}\right)^{2}}{2 \sigma_{\Omega_{p}}^{2}}\right] \mathrm{d} \omega,
\end{aligned}
$$

where $m$ is the shape parameter of the Gamma distribution, $\Gamma(\cdot)$ is the gamma function [9, Eq. 8.310-1], $\xi=\ln (10) / 10, \Omega_{p}$ is the mean squared-envelop, $\mu_{\Omega_{p}}$ and $\sigma_{\Omega_{p}}$ is the mean and standard deviation of $\Omega_{p}$, respectively. The composite squared envelop is well approximated by a single LN Random Variable (RV) as proposed in [10]. Thus, the parameters of the single LN RV are: shape $\mu_{\mathrm{dB}}=\xi[\psi(m)-\ln (m)]+\mu_{\Omega_{p}}$ and log-scale $\sigma_{\mathrm{dB}}^{2}=$ $\xi^{2} \zeta(2, m)+\sigma_{\Omega_{p}}^{2}[10]$. In this case, $\psi(\cdot)$ is the Euler psi function, $\zeta(2, \cdot)$ is the generalized Riemann zeta function [11], $m$ is the shape parameter of the Gamma distribution,

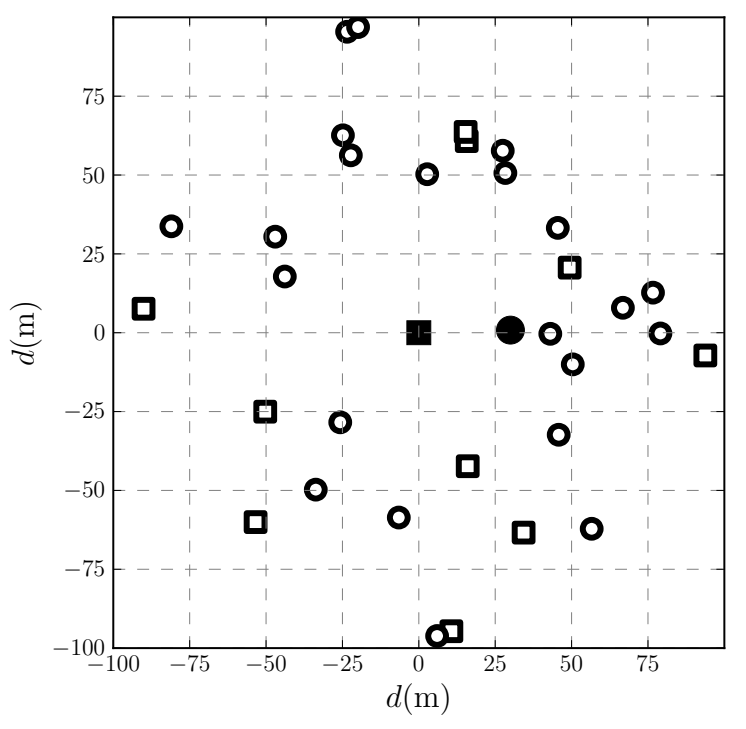

Fig. 1. Illustration of a random deployment of small cells and user terminals over an arbitrary network area. Circles represent user terminals, while squares depict small cell base stations. At the center is depicted the typical pair of BS and UE (filled circle and square).

$\xi=\ln (10) / 10$, and $\Omega_{p}$ is a $\mathrm{RV}$ representing the mean squared-envelope whose mean and standard deviation are given by $\mu_{\Omega_{p}}$ and $\sigma_{\Omega_{p}}$, respectively.

The network deployment model is given by a spatial Poisson Point Processes (PPPs) $\Phi^{\mathrm{BS}}\left(\Phi^{\mathrm{UE}}\right)$, whose random points $\varphi^{\mathrm{BS}}\left(\varphi^{\mathrm{UE}}\right)$ denote the locations of base stations (user terminals). The composite fading channel is associated as random marks to each point of the above processes [12] and is assumed to be independent over distinct network entities and positions. By virtue of the Marking theorem [13], the resulting processes constitute Marked Point Processes (MPPs) on the product space $\mathbb{R}^{2} \times \mathbb{R}^{+}$, is defined as

$$
\widetilde{\Phi}^{\mathrm{BS}}=\left\{(\varphi, x) ; \varphi \in \Phi^{\mathrm{BS}}\right\} .
$$

Note that $\widetilde{\Phi}^{\mathrm{BS}}$ and $\widetilde{\Phi}^{\mathrm{UE}}$ are assumed to be independent spatial PPPs. An annular observation region $\mathcal{O}$, which is respectively delimited by the minimum and maximum radii $R_{m}$ and $R_{M}$, is defined around the user of interest. Fig. 1 illustrates a realization of the random network topology where UEs and small cell BSs are uniformly scattered over network area.

\section{B. Higher Order Statistics and the LN Approximation}

We resort to stochastic geometry in order to analytically model random network deployments [13]-[15], and to higher order statistics to recover both the distributions of the received power $Y$ and the aggregate CCI $Z$ at the user of interest [11], [12]. The Slivnyak's theorem and its associated Palm probability are then used to derive the 
aggregate CCI and compute average performance figures conditional on the location of the user of interest, also known as the "tagged receiver". Next, we apply Campbell's theorem [13] to the MPP $\widetilde{\Phi}$ defined in (3) so as to determine the Characteristic Function (CF) of the distribution of the aggregate $\mathrm{CCI}$.

Definition 1: Let $Z=\sum_{(\varphi, x) \in \widetilde{\Phi}} Y$ be a RV representing the aggregate CCI generated by the interfering process $\widetilde{\Phi}$, and $j=\sqrt{-1}$ be the imaginary unity. Then, the CF of $Z$ is the function $\Psi: \mathbb{R} \rightarrow \mathbb{C}$, which is defined as

$$
\Psi_{Z}(\omega)=\mathrm{E}\left[e^{j \omega Z}\right] \text {. }
$$

The corresponding $n$th cumulants are obtained from higher order derivatives of (4) as shown next [11].

Proposition 1: Let $Z$ be a RV and $\Psi_{Z}(\omega)$ its CF. The $n$th cumulant is denoted by $\kappa_{n}$ where $n \in \mathbb{N}$. Provided that the $n$th moment exists and is finite; then $\Psi_{Z}(\omega)$ is differentiable $n$ times. Therefore,

$$
\kappa_{n}=\frac{1}{j^{n}}\left[\frac{\partial^{n}}{\partial \omega^{n}} \ln \Psi_{Z}(\omega)\right]_{\omega=0}
$$

Proof: See [16, Section 9.4].

Motivated by the fact that the density of $Z$ has no exact closed form expression [17] and that its distribution is heavy-tailed and positively skewed [12], we resort to an approximation of the sum of LN RVs by a single LN RV [17]. The parameters are estimated from the cumulants of the actual distribution of the aggregate CCI as follows,

$$
\mu=\ln \left(\frac{\kappa_{1}^{2}}{\sqrt{\kappa_{1}^{2}+\kappa_{2}}}\right) \text {, and } \sigma^{2}=\ln \left(1+\frac{\kappa_{2}}{\kappa_{1}^{2}}\right),
$$

where $\mu$ is the mean and $\sigma^{2}$ is the variance of the equivalent $\operatorname{Normal}\left(\mu, \sigma^{2}\right)$ distribution in the logarithmic scale.

\section{INTERFERENCE MODEL}

By applying the proposed analytical framework we characterize the resulting aggregate CCI in two Evaluation Scenarios (ESs): ES1 is the benchmark scenario where BSs and UEs operate in HD mode; while in ES2 both BSs and UEs operate in FD mode.

\section{A. Aggregate CCI of a HD network}

Consider the benchmark scenario ES1, we can write the aggregate $\mathrm{CCI}$ at the tagged receiver as

$$
Z_{0}^{\mathrm{HD}}=\sum_{\left(\varphi_{i}, x_{i}\right) \in \tilde{\Phi}^{\mathrm{BS}}} Y_{i 0} .
$$

Notice that the tagged receiver only experiences interference coming from nearby BSs since we assume the DL as the benchmark scenario.

To characterize the aggregate CCI at the tagged receiver, we use the cumulant-based framework with the MPP $\widetilde{\Phi}$
[12], [18] (hereafter we drop the superscript BS). By applying Campbell's theorem to (3), we derive its characteristic functional [13] as given next.

Proposition 2: Consider the ES1; then, the $n$th cumulant of the aggregate CCI perceived by the tagged receiver within $\mathcal{O}$ and with respect to $\widetilde{\Phi}$ is,

$$
\kappa_{n}(\widetilde{\Phi})=\frac{2 \pi \lambda p^{n}}{n \alpha-2}\left(R_{m}^{2-\alpha n}-R_{M}^{2-\alpha n}\right) \mathrm{E}_{X}^{n}[0, \infty]
$$

Proof: See [18, Section V].

\section{B. Aggregate CCI of a FD network}

In the ES2, the tagged receiver is subject to BSs, other UEs and the intrinsic SI component. As a result, the aggregate $\mathrm{CCI}$ at the tagged receiver is characterized by

$$
Z_{0}^{\mathrm{FD}}=\delta p_{00} x_{00}+\sum_{\left(\varphi_{i}, x_{i}\right) \in \widetilde{\Phi}^{\mathrm{BS}}} Y_{i 0}+\sum_{\left(\varphi_{j}, x_{j}\right) \in \widetilde{\Phi}^{\mathrm{UE}}} Y_{j 0},
$$

where $p_{00}$ and $\delta$ represent the SI component and the corresponding attenuation factor, respectively.

To account for the aggregate CCI from multiple interfering tiers we employ the cumulants additivity property. Since BSs and UEs are assumed to be independently deployed, the resulting process from each such tier is also independent [13].

\section{Average Spectral Efficiency}

We evaluate how the FD networks perform in terms of the location-dependent ASE of the tagged receiver [19]. We recall that as discussed in [20] the aggregate interference perceived by the tagged receiver has non-Gaussian nature, and the Shannon formula is used as a lower bound for the ergodic rate. Bearing this in mind and assuming that all users are allocated on the same bandwidth $W$, we first recover the SIR distribution of the tagged receiver, and then compute the respective capacity.

Theorem 1: Under the assumption of the composite fading channel regime, the average spectral efficiency in bits/s/ $\mathrm{Hz}$ of the tagged receiver is given as,

$$
\frac{\bar{C}}{W} \simeq \sum_{k=1}^{K} \frac{\omega_{k}}{\sqrt{\pi}} \log _{2}\left[1+\exp \left(\frac{\eta_{k} \sqrt{2} \sigma+\mu}{\xi}\right)\right] .
$$

Proof: To compute the location-dependent average spectral efficiency, $\frac{\bar{C}}{W}=\int_{0}^{\infty} \log _{2}(1+\gamma) f_{\Gamma}(\gamma) \mathrm{d} \gamma$, we use the PDF of the SIR with respect to the tagged receiver, which is $f_{\Gamma}(\gamma)$. The Gauss-Hermite quadrature [11] with the substitution $\eta=(\xi \ln \gamma-\mu) / \sqrt{2} \sigma$ is used to obtain (10). 


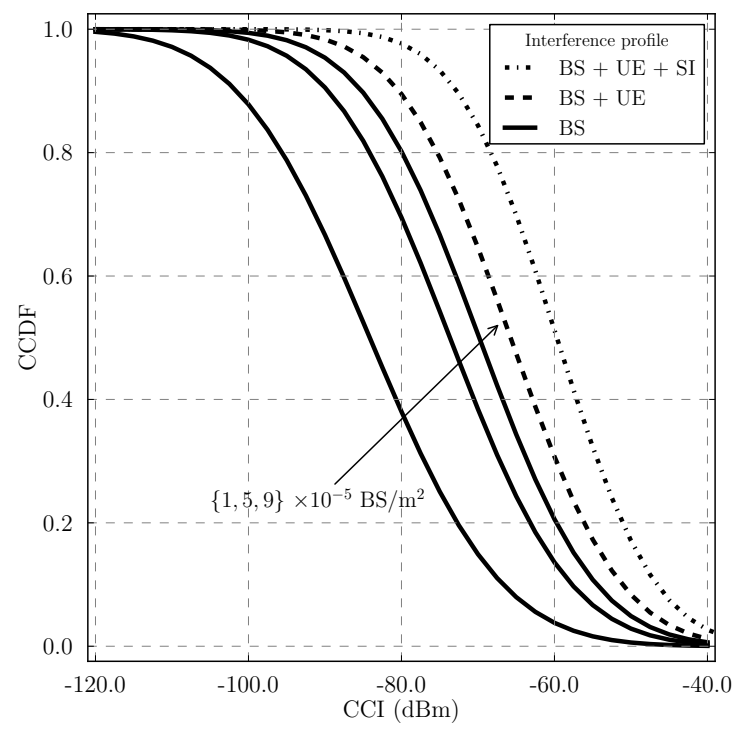

Fig. 2. CCDF of the aggregate CCI at the tagged receiver from an interfering tier of HD BSs (solid lines) for increasing density of interferers. As reference, CCDF of the aggregate CCI ideal FD BSs and practical FD BSs.

\section{NuMERICAL RESUltS}

Next, we present some relevant numerical results ${ }^{1}$. Fig. 2 depicts the Complementary Cumulative Distribution Function $(\mathrm{CCDF})$ of the aggregate $\mathrm{CCI}$ at the tagged receiver for an increasing density interfering small cell BSs (Proposition 2 is used to generate the numerical values). Solid lines present the resulting aggregate $\mathrm{CCI}$ as a function of the increasing density of interfering BSs for the ES1. Note the degradation effect of both the extra interference due to the co-channel UEs as well as to the self-interference. At a density of $10^{-4} \mathrm{BS} / \mathrm{m}^{2}$, we first show the combined interference from co-channel ideal FD BSs and UEs with dashed lines, which represents the best case scenario for ES2. Then, from a more practical point of view of ES2, the total CCI at the FD user of interest for a SI attenuation of $-80 \mathrm{~dB}$ and at the same density of interferers is represented by the dotted line curve. To illustrate our point, the tagged receiver only experiences aggregate $\mathrm{CCI}$ higher than $-60 \mathrm{dBm}$ for about $20 \%$ of the time when in HD mode, whereas the CCI is higher than that value for nearly $50 \%$ of the time in a practical FD setting.

Next, Fig. 3 shows the Cumulative Distribution Function (CDF) of the SIR experienced by the tagged receiver. Notice that the performance is not only degraded by the combined effect of interfering tiers of FD BSs and UEs, but mainly by its own SI component. In this scenario, the SIR of the tagged receiver is severely compromised

\footnotetext{
${ }^{1}$ Unless stated otherwise, assume unitary bandwidth, $\alpha=4, m=2$ which means that all links experience some line of sight, Hermite polynomial order $K=24, R_{m}=25 \mathrm{~m}$ and $R_{M}=250 \mathrm{~m}$.
}

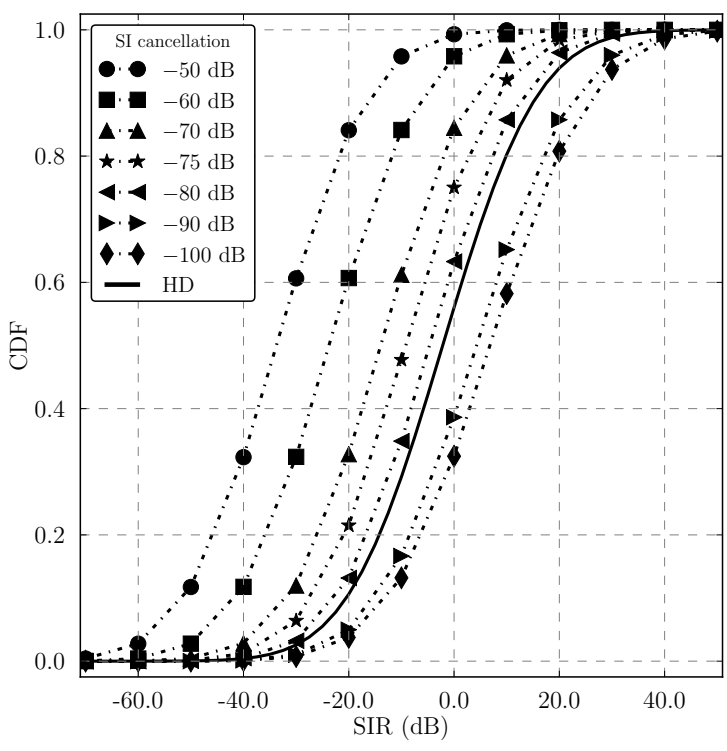

Fig. 3. CDF of the SIR at the tagged receiver from interfering tiers of FD BSs, FD UEs for increasing SI cancellation values.

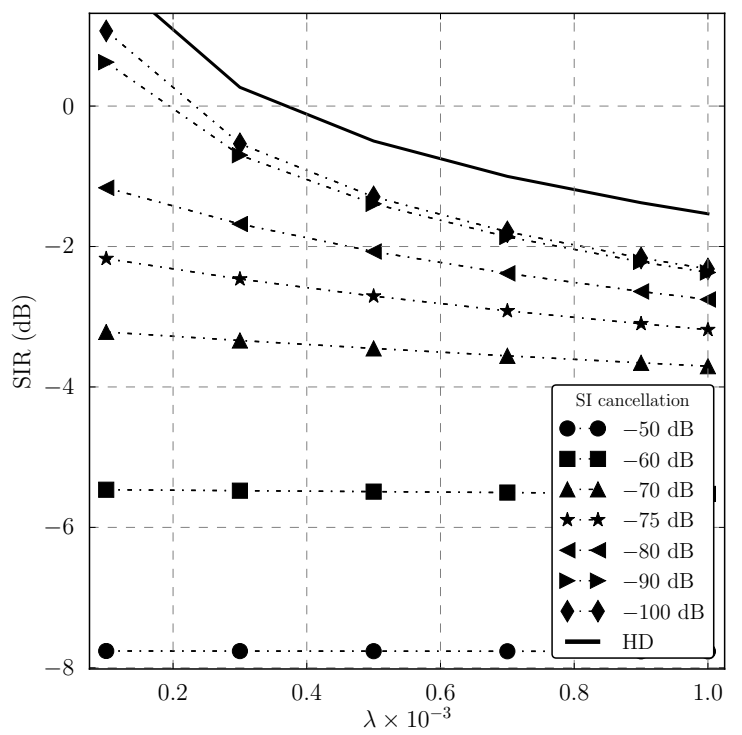

Fig. 4. Average SIR as a function of the density of interferers for different values of SI cancellation $(\delta)$, the SIR of ES1 is shown as reference (solid line)

by the intrinsic SI of FD communications. Notice that as the SI cancellation improves (lower values of $\delta$ ), higher is the perceived SIR. For instance, at $\delta=-75 \mathrm{~dB}$ the SIR values are very low compared to the HD performance (solid line); however, HD and FD perform alike around $\delta=-90 \mathrm{~dB}$ and higher. Nonetheless, we remind that so far experimental results have shown that it is a strenuous task to achieve such high levels of SI attenuation $(\delta<-75 \mathrm{~dB})$ [1]. Therefore, we can conclude that SI dominates the perceived CCI.

Fig. 4 depicts the average SIR as a function of the density of interferers for different values of SI cancellation 
coefficient. Notice that the quality of the SI cancellation scheme employed by the FD UEs considerable affects the perceived SIR. Therefore, as pointed out in [1], [2], the better the SI mitigation, the better the overall performance of FD schemes. Notice that in terms of SIR, HD schemes perceive higher SIR. We recall that contrary to HD schemes, FD communications suffer additional interference from the adjacent BSs besides SI. On the other hand, as we observe in Fig. 5, in terms of ASE FD schemes become more attractive and considerably outperforms the HD scheme even though suffering additional interference. Fig. 5 shows the ASE as a function of density of interferers for different SI cancellation coefficients. For instance, for $\delta=-75 \mathrm{~dB}$ and $\lambda=6 \times 10^{-4}$, FD scheme $50 \%$ higher ASE than the HD schemes, and even larger gains can be achieved if SI cancellation is improved further.

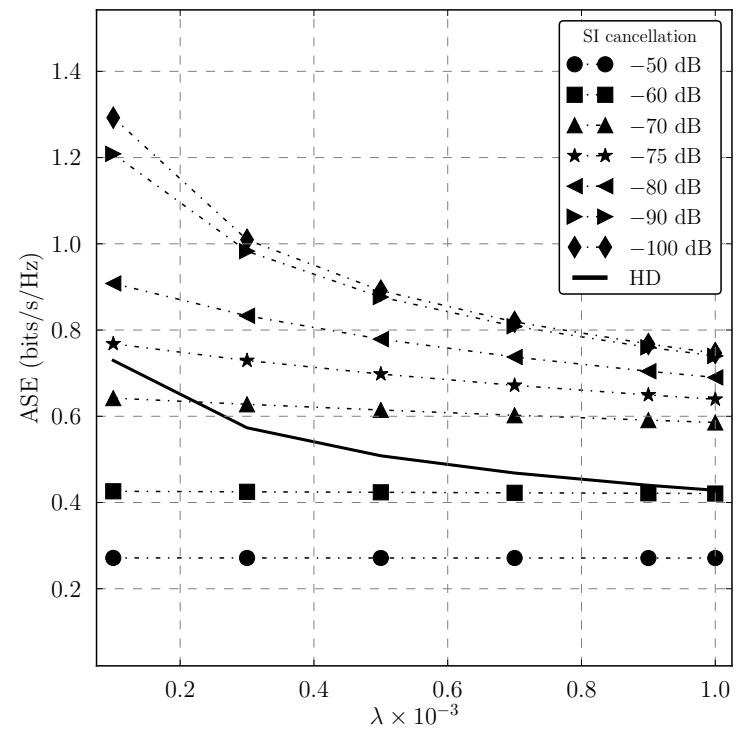

Fig. 5. ASE of ES1 and ES2 as a function of density of interfering nodes. Different SI cancellation coefficients are considered for ES2.

\section{CONCLUSIONS AND FinAl REMARKS}

In this paper, we have assessed the ASE of FD communications in interference networks under composite fading channels. We employed stochastic geometry to model random deployments of BSs and UEs and a composite lognormal shadowing and Nakagami- $m$ fading channels. Our results show that the aggregate interference from both BSs and UEs compromises the FD performance compared to traditional HD networks. Moreover, the SI dominates the aggregate interference component and, in terms of SIR, FD performance becomes comparable to the one achieved in HD mode when the SI cancellation becomes as low as $-90 \mathrm{~dB}$. On the other hand, our results show that, in terms of ASE, FD networks outperform HD networks for SI cancellation values lower than $-70 \mathrm{~dB}(\delta \leqslant-70 \mathrm{~dB})$.

\section{ACKNOWLEDGEMENTS}

Authors would like to thank the Infotech Graduate School, University of Oulu, Academy of Finland and European Union FP7/2007 - 2013 under grant agreement n. 316369 (project DUPLO).

\section{REFERENCES}

[1] M. Duarte, C. Dick, and A. Sabharwal, "Experiment-driven characterization of full-duplex wireless systems," IEEE Trans. Wireless Commun., vol. 11, no. 12, pp. 4296-4307, 2012.

[2] T. Riihonen, S. Werner, and R. Wichman, "Mitigation of loopback self-interference in full-duplex mimo relays," IEEE Trans. Signal Process., vol. 59, no. 12, pp. 5983-5993, 2011.

[3] H. Alves, D. da Costa, R. Souza, and M. Latva-aho, "Performance of block-Markov full duplex relaying with self interference in Nakagami-m fading," IEEE Wireless Commun. Letters, vol. 2, no. 3, pp. 311-314, 2013.

[4] H. Alves, R. Souza, and G. Fraidenraich, "Outage, throughput and energy efficiency analysis of some half and full duplex cooperative relaying schemes," Trans. on Emerging Telecommun. Tech., 2013. [Online]. Available: http://dx.doi.org/10.1002/ett.2658

[5] J. I. Choi, M. Jain, K. Srinivasan, P. Levis, and S. Katti, "Achieving single channel, full duplex wireless communication," in Proceedings of the 16th MobCom, 2010, pp. 1-12.

[6] M. Jain, J. I. Choi, T. Kim, D. Bharadia, S. Seth, K. Srinivasan, P. Levis, S. Katti, and P. Sinha, "Practical, real-time, full duplex wireless," in Proceedings of the 17th MobCom, 2011, pp. 301-312.

[7] H. Ju, S. Lee, K. Kwak, E. Oh, and D. Hong, "A new duplex without loss of data rate and utilizing selection diversity," in IEEE Vehicular Technology Conference (VTC) Spring. IEEE, 2008, pp. 1519-1523.

[8] J. Ilow and D. Hatzinakos, "Analytic alpha-stable noise modeling in a poisson field of interferers or scatterers," IEEE Trans. Signal Process., vol. 46, no. 6, pp. 1601-1611, Jun. 1998.

[9] I. S. Gradshteyn and I. M. Ryzhik, Table of Integrals, Series, and Products, A. Jeffrey and D. Zwillinger, Eds. Academic Press, 2007.

[10] M.-J. Ho and G. L. Stüber, "Capacity and power control for CDMA microcells," ACM Journal on Wireless Networks, vol. 1, no. 3, pp. 355-363, Oct. 1995.

[11] M. Abramowitz and I. A. Stegun, Handbook of Mathematical Functions with Formulas, Graphs, and Mathematical Tables, 9th ed. Dover, 1965.

[12] A. Ghasemi and E. S. Sousa, "Interference aggregation in spectrumsensing cognitive wireless networks," IEEE J. Sel. Areas Commun., vol. 2, no. 1, pp. 41-56, Feb. 2008.

[13] J. F. C. Kingman, Poisson Processes. Oxford Univ. Press, 1993.

[14] P. H. J. Nardelli, M. Kaynia, P. Cardieri, and M. Latva-aho, "Optimal transmission capacity of ad hoc networks with packet retransmissions," IEEE Trans. Wireless Commun., vol. 11, no. 8, pp. 2760-2766, Aug. 2012.

[15] P. H. J. Nardelli, M. Kountouris, P. Cardieri, and M. Latva-aho, "Throughput optimization in wireless networks under stability and packet loss constraints," IEEE Trans. Mobile Comput., vol. PP, no. 99, pp. 1-1, 2013.

[16] S. Resnick, A Probability Path. Birkhäuser Boston, 1999.

[17] J. Wu, N. B. Mehta, and J. Zhang, "A flexible lognormal sum approximation method," in Globecom, St. Louis, MO, 28 Nov.-2 Dec. 2005, pp. 3413-3417.

[18] C. H. M. de Lima, M. Bennis, and M. Latva-aho, "Coordination mechanisms for self-organizing femtocells in two-tier coexistence scenarios," IEEE Trans. Wireless Commun., vol. 11, no. 6, Jun. 2012.

[19] W. C. Y. Lee, "Estimate of channel capacity in rayleigh fading environment," IEEE Trans. Veh. Technol., vol. 39, no. 3, pp. 187189, Aug. 1990.

[20] M.-S. Alouini and A. J. Goldsmith, "Area spectral efficiency of cellular mobile radio systems," IEEE Trans. Wireless Commun., vol. 48, no. 4, pp. 1047-1066, Jul. 1999. 